"This is the peer reviewed version of the following article: Adv. Mater. Interfaces. 2018, 5 (9), 1701585, which has been published infinal form at DOI: 10.1002/admi.201701585. This article may be used for non-commercial purposes in accordance with Wiley Terms and Conditions for Self-Archiving published at http://olabout.wiley.com/WileyCDA/Section/id-820227.html." 


\title{
Enhanced Conductivity for Carbon Nanotube based Materials through Supramolecular Hierarchical Self-Assembly
}

\author{
Gustavo A. Zelada-Guillén, ${ }^{*[a, b]}$ Martha V. Escárcega-Bobadilla, ${ }^{*[a, c]}$ Marcin Wegrzyn, ${ }^{[d]}$ Enrique \\ Giménez, ${ }^{\left[{ }^{[e]}\right.}$ Gerhard Maier ${ }^{[b]}$ and Arjan W. Kleij ${ }^{*[c, f]}$
}

\begin{abstract}
The currently limited comprehension of hierarchical control over out-of-equilibrium (dynamic) self-assembly processes in nanoscience and nanotechnology has limited the exploitation of multicomponent systems in the design of new nanostructured functional materials. In our contribution, molecular building blocks with tailored nanoscale anisotropic supramolecular self-assembly behavior enable the creation of mesoscale percolation networks of multiwall carbon nanotubes through collinear interconnections at the microscale. This strategy affords polymeric composites with tunable properties at the macroscale, where the organization mechanism is regulated by dynamic self-assembly at 4 hierarchical levels of autoorganization. Such multilevel self-assembly system reduces up to 8fold the nanotube concentration required for percolation and enhances conductivity up to 6 orders of magnitude against blanks, thus yielding anisotropically semiconducting and conducting materials. Our approach is based on casting-from-solution, thus simplifying preparative steps when compared to state-of-the-art electron carrier counterparts such as single-walled carbon nanotube-, graphene- or indium-tin-oxide-based technologies. Finally, promising material transparency levels can be reached across the visible and nearinfrared regimes for compositions above the percolation threshold, which provides new opportunities beyond the current spectral restrictions in commercial transparent conductors.
\end{abstract}

\section{Introduction}

The deployment of nanoscale objects into industrially viable multicomponent functional materials requires for the

[a] Dr. G. A. Zelada-Guillén, Dr. M. V. Escárcega-Bobadilla School of Chemistry, National Autonomous University of Mexico (UNAM), Circuito Escolar s/n, Ciudad Universitaria, Mexico City 04510 (Mexico).E-mail: g.zelada@unam.mx, mesbo@unam.mx

[b] Dr. G. A. Zelada-Guillén, Dr. G. Maier; Polymaterials AG, Innovapark 20, Kaufbeuren 87600 (Germany)

[c] Dr. M. V. Escárcega-Bobadilla, Prof. A. W. Kleij Institute of Chemical Research of Catalonia (ICIQ), the Barcelona Institute of Science and Technology (BIST), Av. Paisos Catalans 16 43007 - Tarragona (Spain). E-mail: akleij@iciq.es

[d] Dr. M. Wegrzyn, Instituto Tecnológico del Plástico (AIMPLAS), C. Gustavo Eiffel 4, Paterna 46980 (Spain). Current address: School of Engineering, Engineering Research Institute, Jordanstown campus, Shore Road, Newtownabbey, Co. Antrim, BT37 0QB, Ulster (UK)

[e] Dr. E. Giménez, Instituto de Tecnología de Materiales, Universidad Politécnica de Valencia, Valencia 46022 (Spain)

[f] Prof. A. W. Kleij, Catalan Institute for Research and Advanced Studies (ICREA), Pg. Lluis Companys 23, Barcelona (Spain)

Supporting information for this article is given via a link at the end of the document. simultaneous resolution of complex problems. Those problems include optimal orientation, ordering and distribution of nanostructures, ${ }^{[1]}$ their hierarchical control at multiple scales ${ }^{[2]}$ (e.g. nanoscale, microscale, mesoscale and macroscale) and cost-effectiveness of the raw materials ${ }^{[3]}$ together with simple and scalable processing steps. In this sense, the hierarchical selfassembly of molecular, macromolecular or nanostructured building blocks (BBs) via supramolecular interactions has emerged as a promising strategy that allows for spontaneous control of matter at different scales. Hierarchically organized systems have found many uses including recognition elements in theranostic tools, ${ }^{[4]}$ stimuli-responsive parts in soft materials, ${ }^{[5]}$ transducers and mesoporous components in chemical sensors, ${ }^{[6]}$ hybrid bio-organometallic assemblies in optical switches, ${ }^{[7]}$ multilayer topologies in energy conversion/storage systems ${ }^{[8]}$ and patterned conductors ${ }^{[9]}$ in electronic devices.

However, transparent electrically conducting components in optoelectronic systems still depend on metal oxide technologies (rather than hierarchical self-assembly designs) where indium-tin oxide (ITO) has played a leading role for several decades. ${ }^{[10]}$ Most of the recently explored alternatives in transparent conductors include low-dimensional metal-oxide- or carbon-based electrically conducting nanostructures such as nanoparticles, nanowires, nanorods and nanosheets. ${ }^{[11]}$ Unfortunately, the development of those alternatives by precisely controlling these structures at the nanoscale has remained a difficult task so far. ${ }^{[5,9 c, 12]}$ An interesting alternative is the use of highly-stable, non-conducting transparent polymers (e.g. polymethyl methacrylate, PMMA; polyethylene terephthalate, PET; polystyrene, PS; polycarbonate, PC) as matrices in multicomponent (composite) material films or as substrates for randomly deposited $1 \mathrm{D}$ and $2 \mathrm{D}$ electron carriers such as carbon nanotubes (CNTs) or graphene. ${ }^{[13]}$

In the case of CNTs, the matrix may contribute to a high physical resistance in the film while the CNTs should work as nanoscale 'electrical wires' when they exceed a percolation threshold $\left(p_{\mathrm{c}}\right)$, i.e. the limit beyond which an efficient interconnection between individual electron carriers reaches the dimensions of the system. Nowadays, CNT-based conducting systems rely on single-walled carbon nanotubes (SWCNT) because they are easier to solubilize, offering good conductivity values ( $\sigma$ from $10^{-2} \mathrm{~S}$ to $10^{-3} \mathrm{~S}$ ) and allowing for transparency degrees between $70 \%$ and $90 \%$. The latter performance has not been possible when their yet much less expensive and industrially produced multi-walled counterparts (MWCNT) are used, ${ }^{[12 a, 12 d, 13 b, 14]}$ even if hierarchical topologies (i.e. twisted yarns) ${ }^{[15]}$ are employed.

However, in all the examples using MWCNTs, higher $\sigma$ values or larger percolation networks were only possible if multiple nanotube layers or higher loadings thereof were used. 
Nevertheless, in microscale/mesoscale systems the film dimensions exceed the length of the nanotubes and the last option results unpractical; the higher the loadings the higher the amount of agglomerates which negatively affect percolation at the macroscale. ${ }^{[16]}$ Conversely, electrical percolation in the bulk also depends on the nanotube-nanotube connectivity angle average at the nanoscale, with fewer collinearly interconnections, the lower the volumetric conductivity $\left(\sigma_{v}\right) \cdot{ }^{[17]}$ Unfortunately, the achievement of a balance between collinearity in interconnections at the nanoscale, adequate dispersion at the microscale and preservation of a continuous conductive network at the mesoscale, have remained elusive so far. In a recent work, chemical unzipping of MWCNTs was used to produce nanotubes bridged by graphene nanoribbons in conductive $2 \mathrm{D}$ networks supported on fluorine-doped tin oxide substrates, where percolation depended on the unzipping degree. ${ }^{[18]}$ On the contrary, in solid matrices, percolation among carbon nanostructures depends on stochastic and entropic factors, which has limited the development of high-throughput processes.

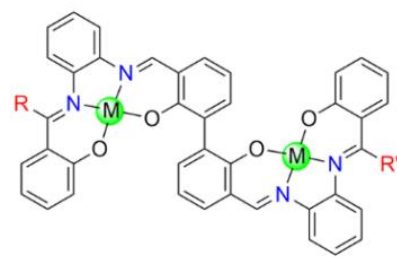

Figure 1. Supramolecular synthon based on a modular tetrakis-Schiff base metal complex able to work as a self-assembly BB. In the structure: $R=R^{\prime}=$ Aromatic, $\mathrm{M}=\mathrm{Cu}(\mathrm{II}), \mathrm{Ni}(\mathrm{II})$ or $\mathrm{Zn}(\mathrm{II})$.

Nature offers examples where supramolecular self-assembly has evolutionary served as the simplest route to overcome such entropic limitations in out-of-equilibrium (dynamic) systems. This can translate, for instance, molecular information into adaptive patterns in information delivery networks such as in the cytoskeleton in eukaryotic cells and neurons in animal nervous systems. Recently, we designed a supramolecular synthon that self-assembles into nanoarchitectures that mimic at the mesoscale webs of neurons in the brain either on surfaces or in polymer matrices by simple casting-from-solution. ${ }^{[19]}$ In these webs, dispersed nanorings (the 'neurons') are interlinked by long slender nanowires (the 'axons'/'dendrites'), where the BB is derived from a modular tetrakis-Schiff base complex (Figure 1). However, in those preliminary studies, the mechanism by which the system could be potentially used as a self-assembly template for creating networks of CNTs in the mesoscale through their relocation from random clusters remained essentially unknown. More particularly the exact nature of the relationship between the nanoscale-controlled microscale self-assembly process of the Schiff base complex and the directed organization of the CNTs on the macroscale properties such as electrical percolation or optical transparency in the UV-VIS-NIR region remained an open question.

In this contribution we describe a detailed mechanistic manifold helping to increase the fundamental understanding of how translating self-assembly principles at different levels of organization and designing new materials that enable the potential of new conducting systems with promising transparency levels.

\section{Results and Discussion}

\section{Mechanism of self-assembly}

For the self-assembly process, we employed two tetrakis-Schiff base metallic complex variants, 1 and $\mathbf{2}$ (which is a version of $\mathbf{1}$ with an extended aromatic surface), in systems meeting the requisites to render the same self-assembly behavior (Figure 2, see supporting information). These facile to prepare and easily modulated $\mathrm{BBs}^{[20]}$ were used to provide a potential solution for the issues of CNT debundling and their collinear interlinking in a polymer matrix through self-organization.

a)
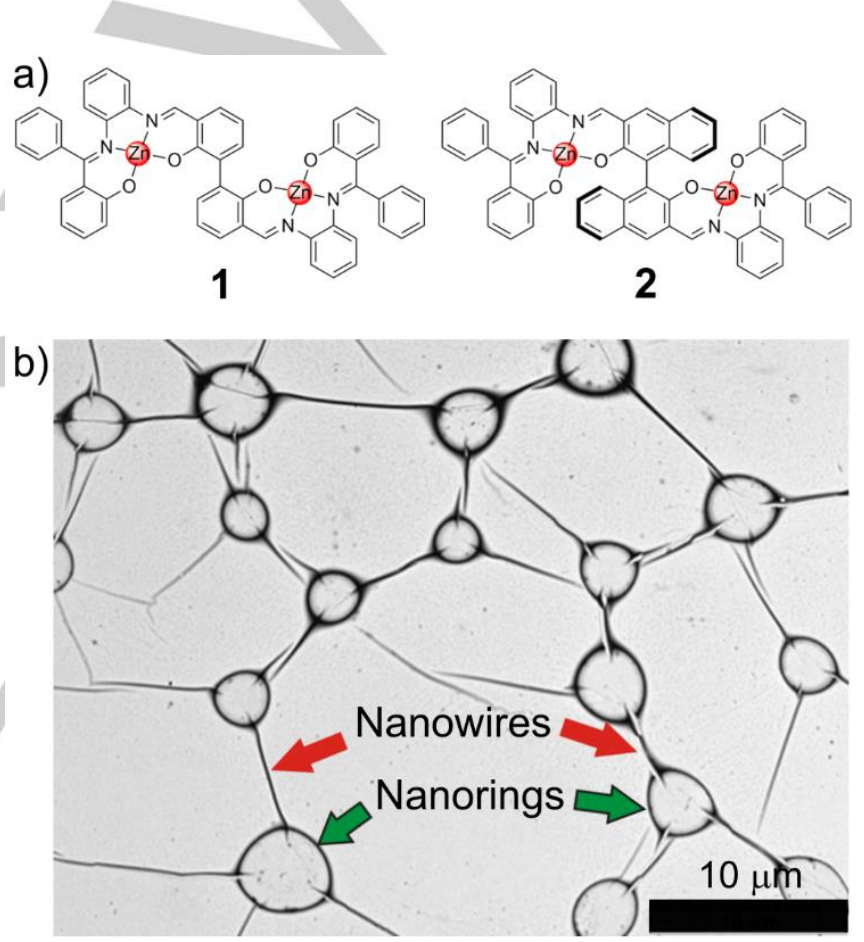

Figure 2. BBs used in this work: a) supramolecular synthons 1 and $\mathbf{2}$, b) network self-assembly behavior observed by TEM for a dropcast solution prepared with $2(70 \mu \mathrm{m})$ in DCM.

Dynamic light scattering (DLS) and transmission electron microscopy (TEM) studies show that the auto-organization of nanotubes is mediated by interfacial mass transfer and energy dissipation during solvent evaporation through hierarchical selfassembly involving four levels of organization.

This multilevel mechanism (Scheme 1) explains the features observed both in drop-cast dichloromethane (DCM) solutions without polymer and in polymer films after solvent evaporation. In a polymer solution, the nanotube bundles are individualized by a superficially self-assembled multilayer of the BBs (level 1) at the 
nanoscale via $\pi-\pi$ interactions and van der Waals forces. During solvent evaporation, the synthon concentration raises to a critical concentration beyond which they kinetically favor the formation of microscale patchy vesicles with nanotubes adhered to their surface by integrative self-assembly (level 2).

During evaporation, the gradual reduction in solvent volume progressively increases concentration of the components. Such a minimization in the continuous medium promotes mutual adhesion of the vesicles until a close-packed arrangement is reached beyond the microscale. Afterwards, minimization in surface tension at the vesicle-solvent interface arrives with their collapse through toroidal intermediaries (circular-shaped topologies without matter in their geometric center). This collapse displaces the nanotubes from the vesicle surface to the regions which should be the precursors for the rings (level 3). At this latter stage, the aggregates of collapsed vesicles give birth to the nanowires via interfacial migration of matter that emerges from the contact points between vesicles. Simultaneously, the same migration and rearrangement of matter displaces neighboring CNTs from the vesicle edges to the nanowires and distributes the nanotubes along the rim of the final rings, favored by the shape anisotropy of the nanotubes. This process simultaneously occurs throughout the whole system thus promoting formation of longrange networks at the mesoscale (level 4).

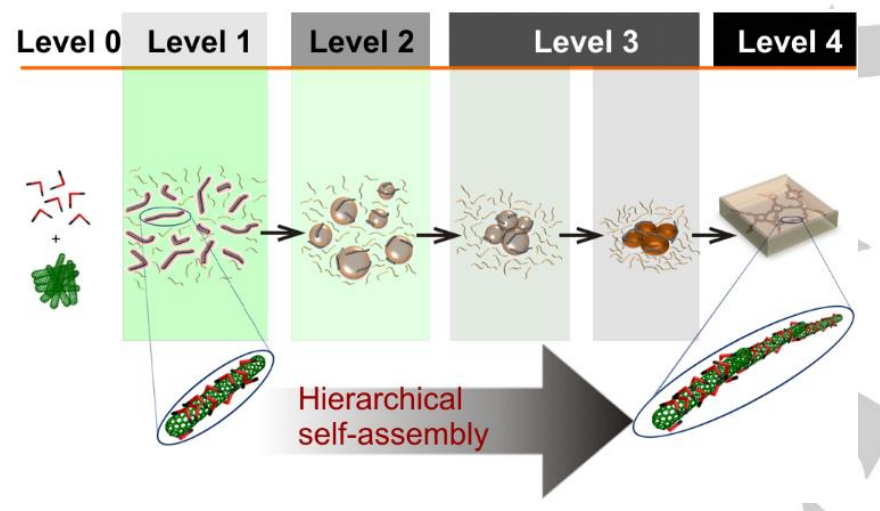

Scheme 1. Mechanism of self-assembly in a polymer solution during solvent evaporation until dryness. Level 0 , BBs in a free form and CNTs in random clusters. Level 1, CNTs covered by a nanoscale self-assembled multilayer of BBs in solution (for clarity purposes, only the closest layer to CNT surface is shown). Level 2, integrated vesicles at the microscale from BBs and CNTs at their surface. Level 3, a large number of intermediary patchy vesicles are in contact beyond the microscale and afford collapsed systems as the solvent evaporates. Level 4, after complete evaporation, an interconnected network of CNTs in a polymer matrix at the mesoscale.

\section{Dynamic self-assembly in solution}

As discussed earlier, the self-assembly of BBs should be related with the evaporative process. Therefore, the type and size of aggregates at different concentrations and compositions would provide useful information to determine the dynamic route towards auto-organization of matter in solution. To study the dynamic self-assembly of the systems, DLS was used to assess the size distribution profiles of aggregates in DCM (Figure 3) for samples containing (i) the BBs $\mathbf{1}$ or $\mathbf{2}$, (ii) the MWCNTs and (iii) their mixtures at different concentrations.

Loss of larger MWCNT agglomerates (at $20 \mu \mathrm{g} / \mathrm{mL}$ ) was observed when $\mathbf{1}$ or $\mathbf{2}$ was present at a $70 \mu \mathrm{M}$ concentration at which long range self-assembled networks can be formed after evaporation with intermediate concentrations between $1 \mu \mathrm{M}$ and $100 \mu \mathrm{M}$. Interestingly, only unimodal size distribution profiles were detected in these mixtures, where the aggregate size values were in the same order of magnitude as found for single nanotubes. The trend suggests the predominance of individual nanotubes in solution and the lack of higher order agglomerates. However, a fourfold increase in the concentration of the previous mixtures at a solvent evaporation degree of $75 \%$ showed a threefold increase in average size of aggregates preserving a unimodal distribution.

Recently, the role of synthon aromaticity of porphyrin oligomers in their supramolecular interactions with the aromatic surface of SWCNTs was spectroscopically demonstrated via $\pi-\pi$ interactions and van der Waals forces. ${ }^{[1]}$ The study was possible taking the advantage of the small size of the nanotubes used and their consequently facilitated solubility in a different solvents. In our systems, both complexes 1 and 2 possess $\pi$-conjugation potential across the ligand framework, while the surface of the MWCNTs are of the same aromatic nature as any of their singlewalled counterparts. Unfortunately, the lack of solubility in DCM of these relatively large nanotubes represent a limitation to further spectroscopic monitoring of BB-CNT interactions. In this regard, DLS analysis is a powerful alternative to the latter, by comparing the aggregate size distributions in BB solutions before and after including the nanotubes, in order to determine whether an association between BBs and CNTs may occur in DCM. When pure BB solutions are analyzed, it is possible to observe that not only large self-assemblies are present (small vesicles, based on further TEM analysis), but also mono-to-oligomolecular entities smaller than ca. $4 \mathrm{~nm}$ occur, which indicates the presence of a dynamic equilibrium between both aggregation types (i.e. intermediate sizes might be kinetically unfavored). After including nanotubes in solution, the smaller entities disappear while the unimodal size distribution mentioned in the previous paragraph is the only observed aggregation, thus indicating a displaced equilibrium towards the association between BBs and CNTs. Taking into account the $\pi$-conjugation in both BBs and nanotubes, a similar interaction phenomena as in Ref. 21 should be expected. Therefore, the observed trend in size regulation of the aggregates governed by the composition of the samples suggests that individualization of nanotubes in solution can be addressed by the aromatic surfaces in the BBs 1 and 2 . This implies that these BBs might interact with the external nanotube walls via interfacial van der Waals and $\pi-\pi$ interactions and this process is dynamically controlled by the evaporation degree. We expect that in future studies, spectroscopic titrations using easier-to-solubilize SCWNTs and similar BBs might be helpful to finally confirm the latter. 

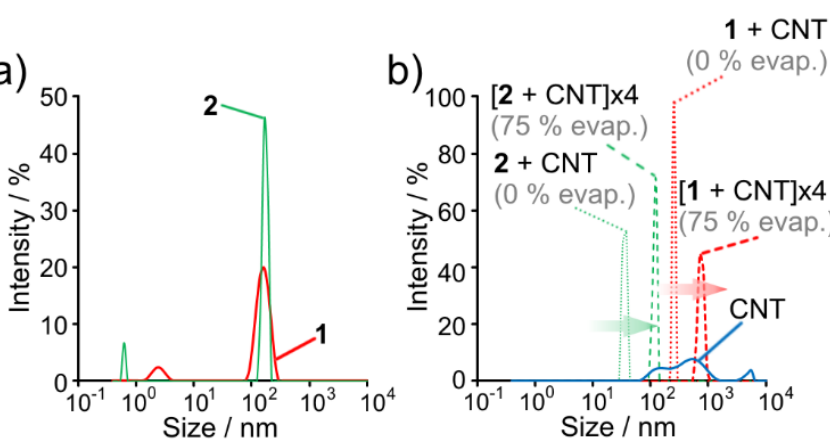

Figure 3. Dynamic self-assembly of BBs $\mathbf{1}$ or $\mathbf{2}$, and MWCNTs in solution. a) Size of aggregates from 1 and 2 in DCM solutions $(70 \mu \mathrm{M})$; b) size distribution of nanotube dispersions at $20 \mu \mathrm{g} / \mathrm{mL}$ in DCM and their counterparts when 1 or 2 are also included at $70 \mu \mathrm{M}$, compared with a threefold increase in size by increasing 4 times the concentration of the pristine mixtures at a solvent evaporation degree of $75 \%$.

\section{Hierarchical auto-organization as a function of solvent evaporation}

The hierarchical self-assembly of the components after evaporation was further studied to evaluate the possibility of dynamical control in size regulation of the aggregates. The same solutions used for the DLS studies were analyzed by transmission electron microscopy (TEM) and compared with samples having different ratios of MWCNT and complex 2 as a model system for the self-assembly mechanism at different evaporation stages. At low MWCNT concentration $(20 \mu \mathrm{g} / \mathrm{mL})$, the BB 2 is involved in a supramolecular wrapping process on the nanotube surface at the nanoscale, where the BB multilayer thickness $(\tau)$ depends on the solution concentration (Figure $4 \mathrm{a}$ ). In this process, the nanotubes are also individualized (Figure $4 \mathrm{~b}$ ) by such nanometer-sized homogeneous interfacial self-assembled multilayer of 2 (hierarchy level 1).
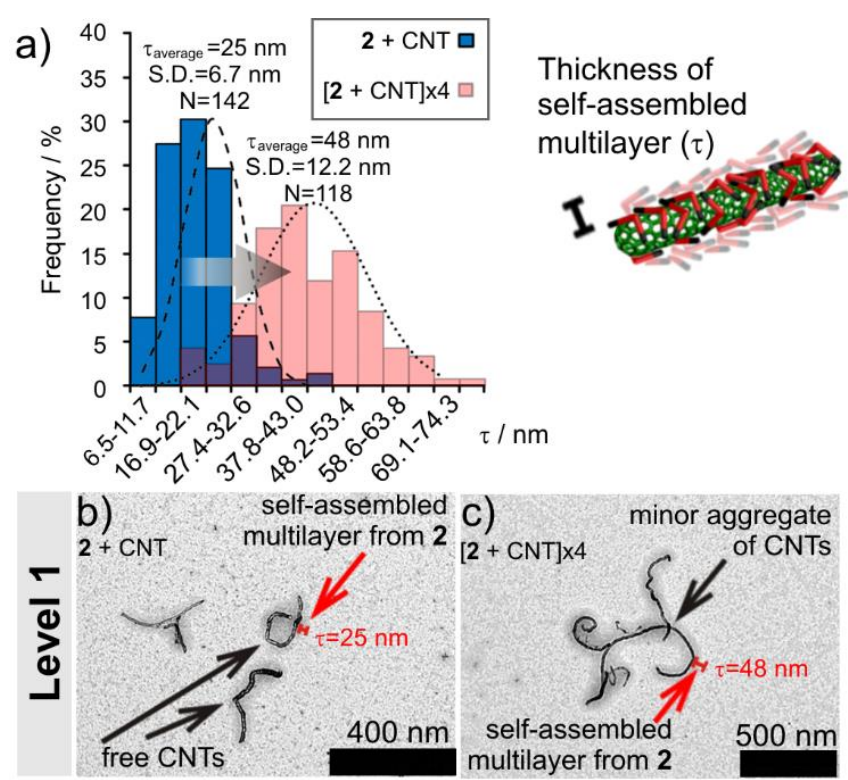

d) three CNTs at the surface $e$ )

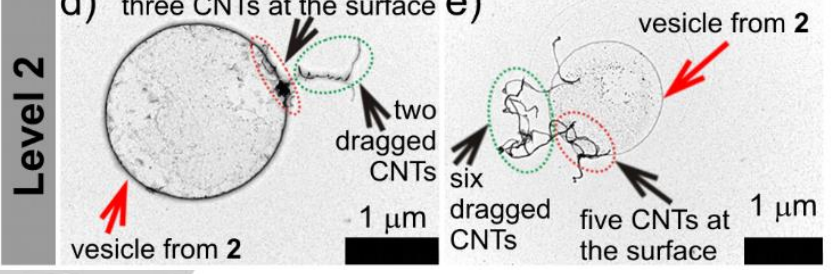

Figure 4. Hierarchy levels 1 and 2 in self-assembly for selected mixtures and the effect of increasing concentration of components. a) In self-assembly level 1 for mixtures containing nanotubes $(20 \mu \mathrm{g} / \mathrm{mL})$ and $2(70 \mu \mathrm{M})$, $\tau_{\text {average doubles }}$ as the concentration of the mixture increases four times: e.g. in the same selfassembly level (b), the self-assembled multilayer of molecule $2(70 \mu \mathrm{M})$ covers the walls of the nanotubes $(20 \mu \mathrm{g} / \mathrm{mL})$ to yield their individualization, while (c) higher concentration of the mixture (fourfold) provides small CNT aggregates with a thicker multilayer of self-assembled 2 (higher $\tau$ ); $d-e$ ) depending on the concentration of nanotubes, self-assembly level 2 becomes more evident, for example, integration of vesicles from $2(70 \mu \mathrm{M})$ is carried out with individual $(\mathrm{d})$ or aggregates (e) of nanotubes ( $20 \mu \mathrm{g} / \mathrm{mL}$ and $80 \mu \mathrm{g} / \mathrm{mL}$, respectively). Panels (b-e) are TEM images of dropcast DCM solutions after evaporation.

A fourfold concentration of a mixture of CNT and BB follows the same trend yet yielding a small number of self-assembled MWCNTs, where each nanotube preserves the superficial multilayer (Figure 4c) with a two-fold increase in its thickness. As the solvent evaporates, the BBs generate vesicles at the microscale (hierarchy level 2) through integrative self-assembly, thus leaving the wrapped nanotubes self-assembled onto the surface. In those vesicles, the number of nanotubes per vesicle is apparently proportional to the concentration of CNTs in the original solution. For example, nanotubes at $20 \mu \mathrm{g} / \mathrm{mL}$ including BB 2 at $70 \mu \mathrm{M}$ produce vesicles occasionally bearing up to three self-assembled nanotubes (Figure 4d). However, solutions with nanotubes having a fourfold of that concentration but the same BB content, generate similar vesicles containing larger numbers of nanotubes (i.e. five self-assembled nanotubes, Figure 4e). Those nanotubes self-assembled to the vesicles can also drag non-self-assembled nanotubes that are mechanically attached to the former ones. This behavior is similar to the mechanism of the 
'hook-and-loop' fasteners (or Velcro® tapes). The nanotubes which are self-assembled to the vesicle surface play the role of the 'hooks', whereas the dragged nanotubes act as the 'loops'. Hence, in solution, the vesicles perform a triple role involving recollection, transportation and dispersion of the nanotubes, which also explains the unimodal distribution profiles observed by DLS.
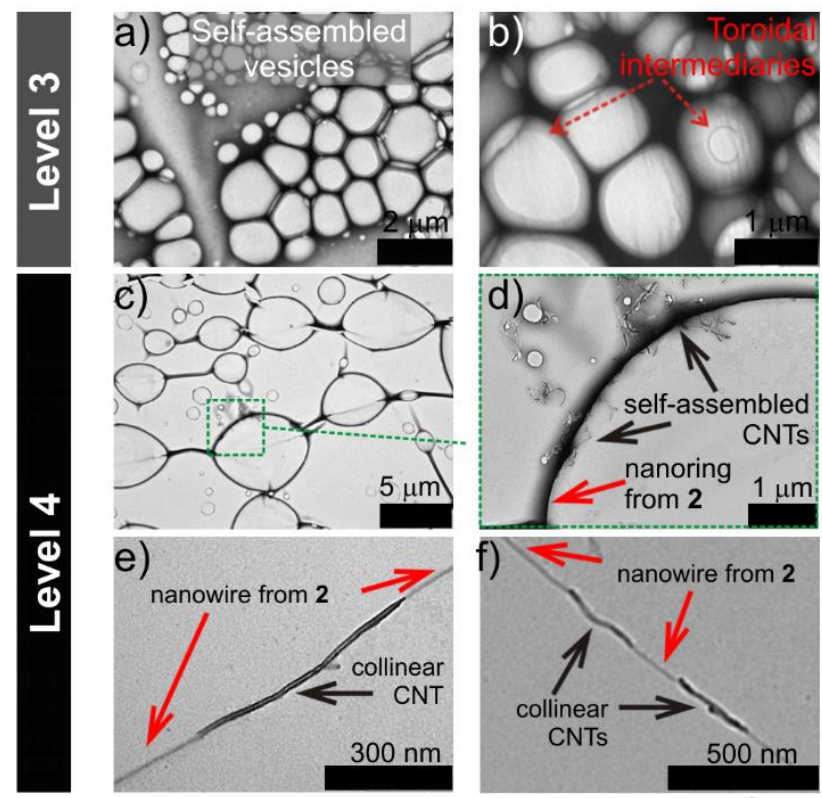

Figure 5. Hierarchy levels 3 and 4 in self-assembly for selected mixtures. Level 3 arises from mutual adhesion of vesicles, which are still organized at level 2 (a), while further relocation of matter at hierarchy level 3 proceeds through toroidal intermediaries (b). At level 4, the network formation addresses a relocation of the nanotube positions to the assemblies where matter from $\mathbf{2}$ is present (c): d) nanorings from 2 contain nanotubes tangentially distributed across the curvature; e-f) nanowires from 2 promote collinear self-assembly of nanotubes. Panels are TEM micrographs from dropcast DCM solutions at selected compositions: 2 at $70 \mu \mathrm{M}(\mathrm{a}-\mathrm{b}), \mathrm{CNTs}$ at $80 \mu \mathrm{g} / \mathrm{mL}$ and 2 at $70 \mu \mathrm{M}$ (cf), (d) is an amplification from dashed box in (c).

Regardless the nanotube concentration in the original solution, solvent elimination simultaneously results into mutual adhesion of a large number of vesicles (previously organized at hierarchy level 2) at larger areas in the microscale (hierarchy level 3, Figure 5a). The same evaporative process results into a collapse in vesicle shape through toroidal intermediaries (e.g. Figure $5 b$ ) and a mass migration process that results in the final network which occurs either in presence or absence of CNTs (see supporting information). During the collapsing of the vesicle, the superficial MWCNTs migrate to the edges to give place to the rim of the rings (Figure 5c-d). Simultaneously, the matter present at the contact points between the vesicles generates nanowires (Figure $5 \mathrm{e}-\mathrm{f}$ ) promoting the reorientation of the nanotubes that remained close to the nanowire-growing points to yield collinear self-assemblies assisted by the anisotropy in shape of both nanotubes and nanowires (hierarchy level 4). The nanowire growth advances as long as the amount of neighboring material allows for an equilibrium point between the final size of the rings and the length of the nanowires. Thus, the relocation of matter evolves until equilibrium is reached between the availability of displaceable material and the mechanical strength of the network, which in optimal conditions may reach mesoscale dimensions. In any case, in the final network (hierarchy level 4), the nanotubes remain covered by the originally self-assembled multilayer of the BB which occurred since earlier stages (e.g. in hierarchy level 1, Figure S2).

In our previous work, ${ }^{[19]}$ DFT and molecular dynamics, together with surface-enhanced Raman scattering microscopy, high-resolution TEM and nanobeam electron diffraction, demonstrated that this type of self-assembled BBs were anisotropically oriented along the longitudinal direction of the nanowires. Those studies also revealed that, on the contrary, BBs were radially auto-organized to the center of the nanorings and concentrically arranged to their rim. Nevertheless, stabilization of these two architectures in the network was possible by four types of intermolecular orientations upon self-assembly. These molecule-to-molecule orientations, namely, back-to-back, stacked, face-to-face and side contact, were derived from the high aromaticity and wedge shape in the BBs and also stabilized the formation of vesicles and oligomers in solution. In the present study, we speculate that those four intermolecular orientations may coexist at each hierarchy level and are responsible for the stabilization of the interconnected nanotubes via $\pi$ interactions at hierarchy level 4 after evaporation. Moreover, the same oriented associations would also explain the favored presence of two particle sizes for BBs in DCM (Fig. 3a), stable oligomers and nanosized vesicles, where the last ones were also detected by TEM. In this sense, the variation in $\tau$ observed by TEM for the superficial multilayer of $\mathrm{BB}$ on the nanotubes at different concentrations (Fig. $4 \mathrm{a}-\mathrm{C}$ ) and the extinction of mono-tooligomolecular entities in solution observed by DLS for BB+CNT mixtures (Fig. 3), may be explained by a nucleation mechanism occurring at the beginning of level 1 . In this process, the highly aromatic surface of the nanotubes should play the role of a nucleation site for self-assembly with free/oligomerized BBs, while this preliminary step may facilitate further association of additional molecules to earlier layers of the same, which in turn, yield the observed multilayer. Interestingly, the growth observed for the BB multilayer as a function of concentration is coherent with the previous hypothesis and we expect that further spectroscopic studies with single-walled counterparts may also confirm this route.

\section{Self-assembly at the micro and mesoscale in} multicomponent materials.

The self-assembly in multicomponent systems was then assessed using the previous casting-from-solution approach applied in the preparation of polymer composite films with a nominal thickness $d$ of $100 \mu \mathrm{m}$. This was done to assess implications of the micro and mesoscale topologies after the selfassembly process on the macroscale properties such as electrical percolation and optical transparency in further evaluations. The films were prepared in combinatorial compositions containing one of the BBs 1 or $\mathbf{2}$ and nanotubes at different concentrations. Therefore, first stock DCM solutions used during the casting 
procedure were prepared by including the selected BBs at the same concentration as the experimental counterparts in the absence of polymer.

Bis-phenol-A based polycarbonate $(10 \% \mathrm{~m} / \mathrm{v})$ was introduced into those stock solutions as a transparent polymer matrix model. After solvent evaporation, solid samples with a nominal content of $\mathrm{BB}$ equal to $0.065 \%$ wt $( \pm 0.005 \%$ wt) were obtained. A microstructural analysis of the films (Figure 6) showed that the BBs simultaneously promoted the collinear orientation at the nanoscale and interconnection of nanotubes at the microscale along the nanowires, similarly to Figure $5 \mathrm{e}-\mathrm{f}$. These features were found by TEM regardless of the direction in which the material was analyzed (e.g. transversal vs. longitudinal internal sections in Figure $6 \mathrm{c}$ and $6 \mathrm{~d}$, respectively).

This trend confirms the creation of interconnected nanotube networks at the microscale throughout the bulk and opens an opportunity to modulate not only $\sigma_{v}$ but also surface conductivity $\left(\sigma_{s}\right)$ in the materials. On the contrary, blank samples which only included nanotubes displayed random CNT bundles in the bulk (Figure 6a). The trend observed by TEM at the nanoscale and microscale inside the materials was consistent with the surface features respectively observed by scanning electron microscopy (SEM) on the native films without (Figure 6e) or with BB (Figure $6 f)$, thus confirming this behavior at the mesoscale too.
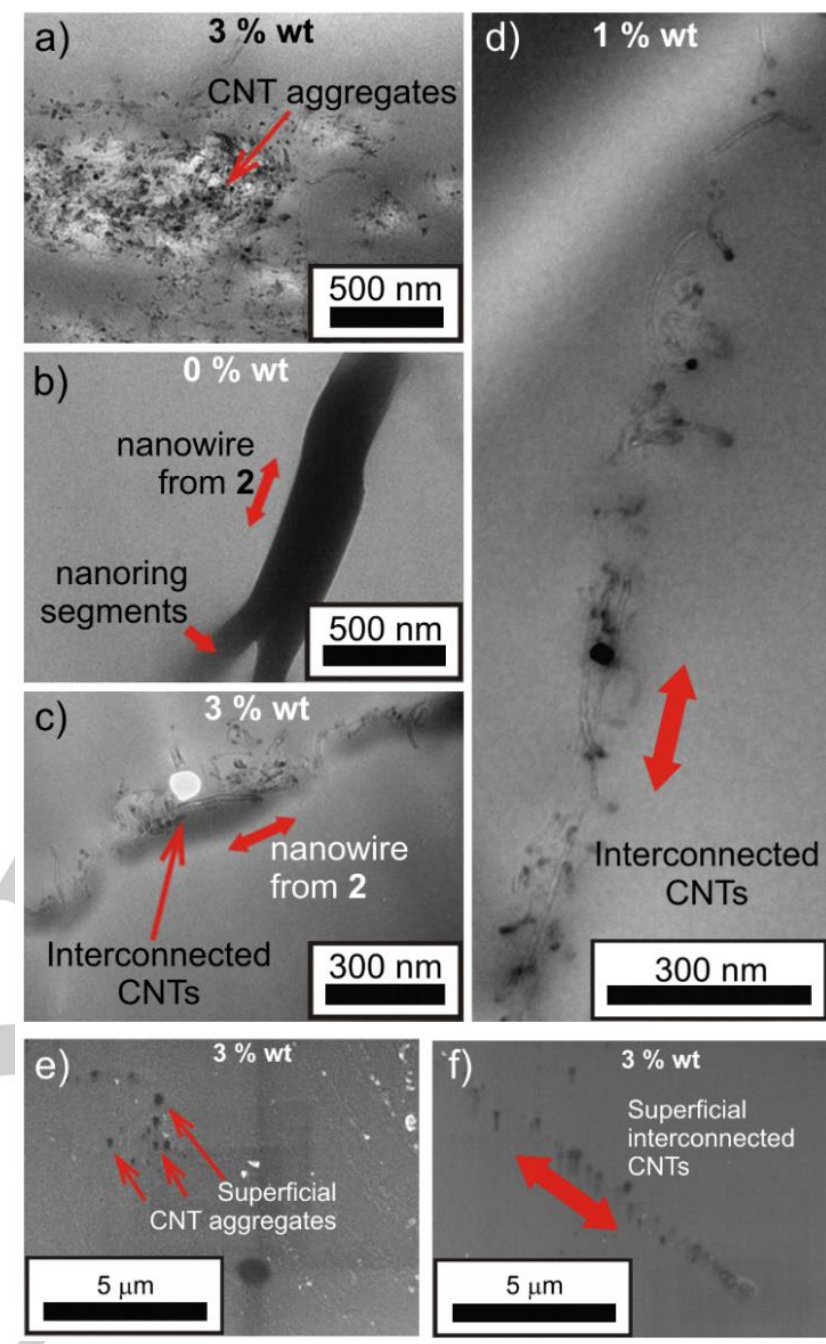

Figure 6. Structural analysis of polymer films at different compositions. Panels (a-d) are TEM micrographs from internal sections in the PC films. a) Transversal cut from a blank film with CNTs at $3 \%$ wt; b) transversal section from a film with 2; c) transversal cut view from a sample containing 2 and CNTs $3 \%$ wt; d) longitudinal cut from a specimen with 2 and $1 \%$ wt nanotubes. Panels (e-f) are SEM micrographs from the surface of selected films. e) Blank film with nanotubes at $3 \% \mathrm{wt} ; \mathrm{f}$ ) film containing BB 2 and CNTs at $3 \%$ wt.

\section{Macroscale functional properties in films.}

The macroscale properties of the multicomponent materials were determined by variation of film compositions using a wide range of nanotube concentration ( $1 \times 10^{-4} \%$ wt to $\left.5 \% \mathrm{wt}\right)$. Preliminarily, analysis of specimens at the latter compositions demonstrated the possibility to modulate the electrical properties. Film samples were electrically tested to define the trend on $\sigma_{v}$ and $\sigma_{s}$ as a function of the nanotube concentration in self-assembled and non-self-assembled scenarios. Assessment of $\sigma_{v}$ (Figure 7a) demonstrated that the hierarchical self-assembly process induced by either $\mathbf{1}$ or $\mathbf{2}$ at the nano, micro and mesoscale, promoted close to a two-fold decrease in the nanotube concentration at which percolation begins in control experiments $\left(p_{\mathrm{c}}, 0.079 \% \mathrm{wt}\right.$ and $0.096 \%$ wt for 1 and 2 versus $0.169 \%$ wt for the blank, respectively). 
a)

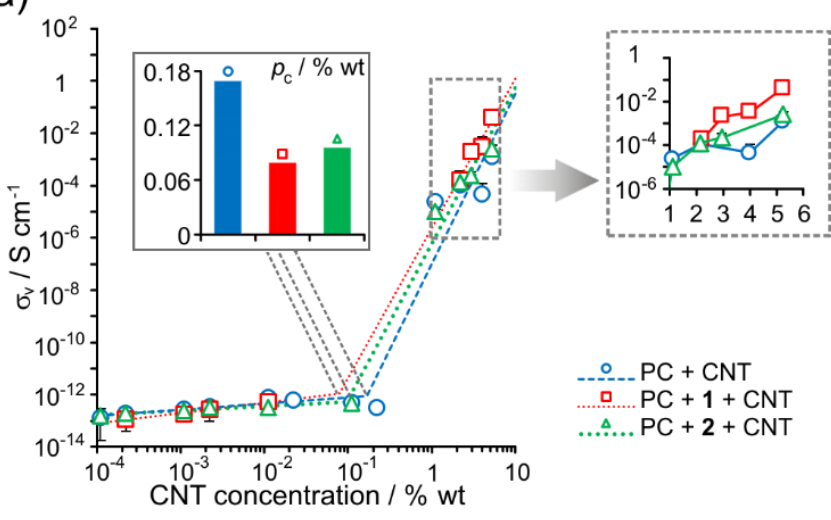

b)

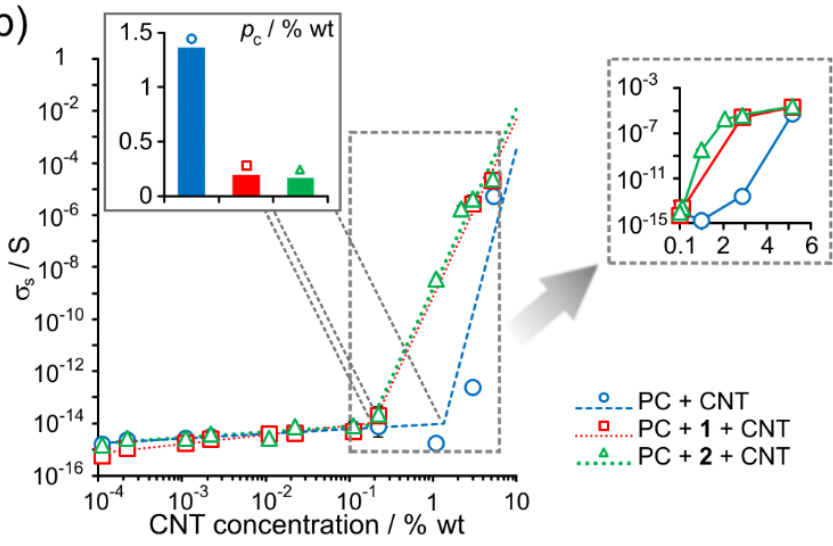

c)

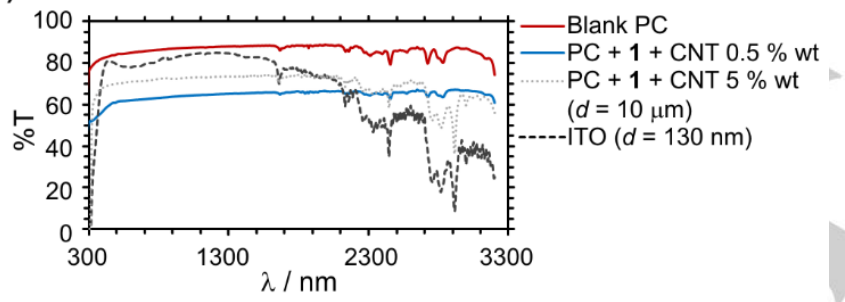

Figure 7. Electrical and optical properties of films. Panels (a-b) show the effect of CNT concentration (from $10^{-4} \%$ wt to $5 \% \mathrm{wt}$ ) and BBs 1 or 2 on $\sigma_{v}(\mathrm{a})$ and $\sigma_{\mathrm{s}}(\mathrm{b})$ for a selected $d=100 \mu \mathrm{m} ; p_{\mathrm{c}}$ are indicated at intersection of trend lines and plotted as bar chart insets; insets in dashed squares are magnifications from the indicated areas; error bars for $(a-b)$ are $1 \mathrm{SD}$ for $\mathrm{N}=3$. c) Combined effect from CNT concentration $(0.5 \%$ wt $v s .5 \%$ wt) and film thickness $(d=100$ $\mu \mathrm{m} v$ s. $d=10 \mu \mathrm{m}$ ) for selected BB 1 compared to ITO with $d=130 \mathrm{~nm}$ and a standard blank PC sample with $d=100 \mu \mathrm{m}$; in (c), ITO and film specimen with $d=10 \mu \mathrm{m}$ are deposited on a $d=50 \mu \mathrm{m}$ PET film.

On the other hand, the same outcome was observed evaluating $\sigma_{\mathrm{s}}$ (Figure $7 \mathrm{~b}$ ), where the BBs triggered an 8-fold reduction in the percolation threshold concentration as opposed to non-selfassembled equivalents (respectively, $p_{\mathrm{c}} 0.174 \%$ wt and $0.198 \%$ wt for 2 and 1 versus $1.363 \%$ wt for the blank).

In addition, self-assembled systems prepared above the percolation threshold concentration raised the $\sigma_{v}$ values between 1-to-3 orders of magnitude as compared to blank counterparts, where the conductivity values reached up to $10^{-5} \mathrm{~S} \cdot \mathrm{cm}^{-1}$ to $10^{-1}$ $\mathrm{S} \cdot \mathrm{cm}^{-1}$ between $1 \% \mathrm{wt}$ and $5 \% \mathrm{wt}$, respectively. In contrast, self- assembled systems afforded significant improvement in $\sigma_{\mathrm{s}}$ over their respective blanks above the percolation threshold. The selfassembly process enhanced $\sigma_{\mathrm{s}}$ of about $6-7$ orders of magnitude for CNT concentrations between $1 \%$ wt and $3 \%$ wt up to around $10^{-6} \mathrm{~S}$ to $10^{-5} \mathrm{~S}$ for loadings beyond $2 \%$ wt while higher nanotube contents inhibited this effect.

Such a difference in the trend observed for $\sigma_{s}$ compared to $\sigma_{v}$ is evidence that not only the self-assembly mechanism provides macroscale conductivity but it also means that anisotropic percolation is reached at the microscale and mesoscale. This anisotropic behavior might be a result of a higher density of the number of conductive paths and more extensive lengths along the surface of the prepared films at the mesoscale in contrast with a lower density of the number of continuous interconnections from one face of the sample to the opposite one in the microscale. The latter is also coherent with both the surface features detected by SEM and the internal structures observed by TEM from samples prepared at concentrations beyond the percolation threshold.

Visible and NIR are appealing regions for potential applications in energy conversion systems such as solar cells. In this sense, the NIR range is still underexploited in commercial devices. For comparison purposes, in the form of a thin film (ca. $10^{2} \mathrm{~nm}$ ), standard transparent conductors such as ITO present an acceptable balance between electrical conductivity $\left(\sigma \mathrm{ca} \cdot 10^{-2}\right.$ to $10^{-1} \mathrm{~S}$ ) and optical transmittance mostly in the visible range (\%T around $80 \%$ ), whereas it is opaque in the NIR region. ${ }^{[11,22]}$ Therefore, the assessment of the optical transmittance of the previous films across the entire UV-VIS-NIR range would represent an interesting starting point to search for potential substitutes for ITO in the future. Both BBs 1 and 2 showed a negligible effect on the optical properties in the VIS-NIR as their signals remained irrelevant in comparison with those from the polymer matrix (Figure S3). Moreover, the nanotubes did not contribute with additional absorption peaks between $500 \mathrm{~nm}$ and $3200 \mathrm{~nm}$, yielding flat spectral regions, but average transmittance $(\% \mathrm{~T})$ was reduced down to $\mathrm{ca}$. $60 \%$ for CNT loadings above $0.5 \%$ wt.

However, modulation of the film thickness $d$ should help to control transparency according to the Lambert-Beer law, as the thicker the film the lower the transparency at a selected wavelength at equivalent compositions. Therefore, nominal $d$ was evaluated at two levels $(100 \mu \mathrm{m}$ and $10 \mu \mathrm{m})$ whereas the nanotube content was incorporated at $0.5 \%$ wt and $5 \%$ wt (Figure 7c) using 1 as a model system. The results showed that thinner films with a higher nanotube loading afforded higher transparencies from $400 \mathrm{~nm}$ to $2700 \mathrm{~nm}$ when compared to thicker films with a lower nanotube concentration. This confirmed that it is possible to control transparency in self-assembled systems by modulating the film thickness. Interestingly, both selfassembled systems operated much more competitively at $\lambda>$ $2100 \mathrm{~nm}$ than ITO films with $d=130 \mathrm{~nm}$, while films with CNTs at $5 \%$ wt and $d=10 \mu \mathrm{m}$ showed comparable transparency levels to ITO in the range 1700-2100 $\mathrm{nm}$ and acceptable transparency values in the range $400-1700 \mathrm{~nm}$. Therefore, the transparency was measured at a standard $\lambda=550 \mathrm{~nm}\left(\% \mathrm{~T}_{550}\right)$ for samples with different nanotube loadings at the two nominal $d$ values for both BBs (Figure S4). 
In both $d$ versions, film transparency dropped exponentially as the nanotube concentration was stepwise increased from $10^{-4} \%$ wt to $5 \%$ wt. The samples with $d=10 \mu \mathrm{m}$ afforded the lowest impact on the transparency across the whole range, making it possible to achieve very competitive $\% \mathrm{~T}_{550}$ values between $70-100 \%$ for the whole loading range. On the other hand, the thicker films were only competitive when the CNT loading is kept below $1 \%$ wt. Interestingly, the self-assembly process produced a weak decrease on $\% \mathrm{~T}_{550}$ for nanotube loading above $0.2 \%$ wt in comparison with blanks. This effect may be attributed to three factors. First, compared to the blanks, a reduction in CNT agglomerates by self-assembly should homogenize the number of nanotubes per unit area and decrease the void zones that are exposed to light in these tests. Second, when $\mathbf{1}$ or $\mathbf{2}$ are present, they should promote an increase in the number of interconnected nanotubes in the material thus establishing tridimensional networks that at larger nanotube loading imply thicker selfassembled structures (cf., thinner self-assemblies at $1 \% \mathrm{wt}$ in Figure $6 \mathrm{~d}$ versus thicker interconnections at $3 \% \mathrm{wt}$ in Figure $6 \mathrm{c}$ ). Third, the combined effect of the previous two factors should result into smaller relative standard deviation in average $\% \mathrm{~T}_{550}$ when measured in the self-assembled films above the percolation threshold. The three previous suppositions were consistent with the combined DLS, TEM and SEM observations as well as with $\% \mathrm{~T}_{550}$ for the whole nanotube loading range.

Finally, one of the most remarkable contributions from our study is that a percolation network is reached through hierarchical self-assembly at the nanoscale, microscale and mesoscale which enables the regulation of macroscale properties such as electrical conductivity at very competitive values in semiconducting and conducting regimes $\left(10^{-5} \mathrm{~S} \cdot \mathrm{cm}^{-1}\right.$ to $\left.10^{-1} \mathrm{~S} \cdot \mathrm{cm}^{-1}\right)$. The preliminary results on promising optical transparency in the visible range and superior optical properties in the NIR region using MWCNTs for the first time represent an appealing starting point for future developments using scalable multistage auto-organization.

\section{Conclusions}

In conclusion, this work has demonstrated that the bioinspired nanoscale self-assembly of supramolecular BBs derived from simple and modular Schiff base compounds can be translated to a microscopic control of hierarchically auto-organized and collinearly interlinked MWCNTs to yield mesoscale percolation networks in a polymer matrix. Consequently, those networks converted the polymer system into a functional multicomponent material with tunable, anisotropic electrical conductivity throughout semiconducting and conducting regimes at the macroscale. The mechanism of auto-organization is driven through energy-dissipative processes and multilevel assembly steps. Moreover, the optical performance observed through the visible and NIR regions opens new opportunities beyond the current spectral restrictions observed for commercial transparent conductors, thus paving the way towards a new approach for the generation of conductive materials under scalable and attractive conditions.

\section{Experimental Section}

Synthesis and characterization of chemical compounds: Synthesis of all compounds and their characterization by elemental analysis, Matrixassisted laser desorption/ionization-Time of flight (MALDI-TOF) and High Resolution Mass Spectrometry, ${ }^{1} \mathrm{H}$ and ${ }^{13} \mathrm{C}$ Nuclear Magnetic Resonance $\left({ }^{1} \mathrm{H}\right.$ NMR and $\left.{ }^{13} \mathrm{C} N M R\right)$, and ${ }^{13} \mathrm{C}\left\{{ }^{1} \mathrm{H}\right\}$ Distortionless Enhancement by Polarization Transfer (DEPT) are described below. All starting materials were purchased from commercial sources and used without further purification. Elemental analyses were performed at the Unidad de Análisis Elemental of the University of Santiago de Compostela (Spain). All NMR measurements were carried out on a Bruker- $400 \mathrm{MHz}$ spectrometer at ambient temperature unless stated otherwise, and chemical shifts are given in parts per million versus tetramethylsylane (TMS). Mass spectrometric data were obtained from the Research Support Unit of the ICIQ and MALDI-TOF experiments were carried out with pyrene as matrix. Compounds $(E)-2-\left(\left((2-a m i n o p h e n y l)\right.\right.$ imino)(phenyl)methyl)phenol, ${ }^{[23]} 3,3^{\prime}-$ diformyl-2,2' -dihydroxy-1,1'-biphenyl, ${ }^{[24]}$ 2-hydroxy-[1,1'-biphenyl]-3carbaldehyde, ${ }^{[25]} \quad 3,3^{\prime}$-bis $((E)-((2-((E)-((2-$ hydroxyphenyl) $)$ (phenyl)methylene)amino)phe-nyl)imino)methyl)-[1,1'-biphenyl]-2,2'-diol (ligand portion of 1 ), and complex $1^{[26]}$ were prepared using previously reported methodologies.

(S)-3,3'-bis((E)-((2-((E)-((2-hydroxyphenyl)(phenyl)methylene)amino) phenyl)imino) methyl)-[1,1'-binaphthalene]-2,2'-diol (ligand portion of 2): (E)-2-(((2-aminophenyl)imino)(phenyl)methyl)phenol (200 mg, 0.7 $\mathrm{mmol}$ ) in $2 \mathrm{~mL}$ of $\mathrm{CHCl}_{3}$ and (S)-2,2'-dihydroxy-[1,1'-binaphthalene]-3,3'dicarbaldehyde (119 mg, $0.35 \mathrm{mmol}$ ) in $5 \mathrm{~mL}$ of $\mathrm{MeOH}$ were refluxed for $24 \mathrm{~h}$. The precipitate formed was then filtered off. Yellow solid, $300 \mathrm{mg}$, 97\%. $[\alpha]_{D}{ }^{25}=-36.37 \mathrm{deg} \mathrm{cm}{ }^{3} \mathrm{~g}^{-1} \mathrm{dm}^{-1}\left(c=0.84 \mathrm{~g} \mathrm{~cm}^{-3}, \mathrm{CH}_{2} \mathrm{Cl}_{2}\right) .{ }^{1} \mathrm{H} \mathrm{NMR}$ $\left(400 \mathrm{MHz}, \mathrm{CDCl}_{3}\right): \delta=6.28\left(22, \mathrm{JHH}_{\mathrm{HH}}=7.8,1.4 \mathrm{~Hz}, 1 \mathrm{H}\right), 6.42\left(\mathrm{td}, \mathrm{J}_{\mathrm{HH}}=7.7\right.$, $1.3 \mathrm{~Hz}, 1 \mathrm{H}), 6.55(\mathrm{~m}, 2 \mathrm{H}), 6.69\left(\mathrm{dd}, \mathrm{JHH}_{\mathrm{HH}}=7.9,1.2 \mathrm{~Hz}, 1 \mathrm{H}\right), 6.76(\mathrm{~m}, 1 \mathrm{H})$, 6.84-7.39 (m, 26H), $7.84\left(\mathrm{~d}, \mathrm{JHH}_{\mathrm{HH}}=8 \mathrm{~Hz}, 2 \mathrm{H}\right), 7.95(\mathrm{~s}, 2 \mathrm{H}), 8.51(\mathrm{~s}, 2 \mathrm{H})$, $12.36(\mathrm{~s}, 2 \mathrm{H}), 14.06(\mathrm{~s}, 2 \mathrm{H}) .{ }^{13} \mathrm{C} \mathrm{NMR}\left(125 \mathrm{MHz}, \mathrm{CDCl}_{3}\right): \delta=117.88$, $118.00,119.50,120.17,121.44,121.57,123.56,123.64,124.88,125.03$, 125.67, 126.99, 127.75, 128.00, 128.79, 129.02, 129.04, 132.31, 133.29, 133.38, 133.50, 134.59, 135.03, 135.09, 135.79, 140.54, 154.44, 154.95, 162.61, 163.92. ${ }^{1} \mathrm{H}$ NMR spectrum, and ${ }^{13} \mathrm{C}\left\{{ }^{1} \mathrm{H}\right\}$ (DEPT) spectra can be found in Figure S5 and Figure S6, respectively. High-resolution mass spectrometry (TOF ES $\left.{ }^{+}\right) \mathrm{m} / \mathrm{z} 883.3326([\mathrm{M}+\mathrm{H}]) \mathrm{C}_{60} \mathrm{H}_{43} \mathrm{~N}_{4} \mathrm{O}_{4}$ requires 883.3284 .

Tetrakis-Schiff base complex 2: To a solution of ligand (S)-3,3'-bis((E)((2-((E)-((2-hydroxyphenyl)(phenyl)methylene)amino)phenyl)imino)-

methyl)-[1,1'-binaphthalene]-2,2'-diol (100 mg, $0.13 \mathrm{mmol})$ in $6 \mathrm{~mL}$ of anhydrous tetrahydrofuran (THF), ZnEt $20.29 \mathrm{~mL} 1 \mathrm{M}$ in hexanes, 0.29 $\mathrm{mmol}$ ) were slowly added. The reaction was stirred at room temperature $18 \mathrm{~h}$. The red precipitate was filtered off. Red solid, $127 \mathrm{mg}, 97 \%$. ${ }^{1} \mathrm{H}$ NMR $\left(400 \mathrm{MHz}\right.$, DMSO- $\left.d_{6}\right): \delta=6.16(\mathrm{~m}, 1 \mathrm{H}), 6.44(\mathrm{~m}, 3 \mathrm{H}), 6.77-7.38(\mathrm{~m}, 26 \mathrm{H})$, $7.70\left(\mathrm{~d}, \mathrm{JHH}_{\mathrm{HH}}=8.1 \mathrm{~Hz}, 2 \mathrm{H}\right), 7.76\left(\mathrm{~d}, \mathrm{JHH}_{\mathrm{HH}}=7.5 \mathrm{~Hz}, 2 \mathrm{H}\right), 8.22(\mathrm{~s}, 2 \mathrm{H}), 9.26(\mathrm{~s}$, 2H). ${ }^{13} \mathrm{C}$ NMR $\left(125 \mathrm{MHz}\right.$, DMSO- $\left.d_{6}\right): \delta=112.15,117.18,119.86,120.58$, 123.14, 123.25, 123.53, 123.96, 124.42, 125.08, 125.71, 126.27, 127.35, $128.13,128.21,128.33,128.50,128.92,129.00,132.99,134.17,137.03$, $137.75,137.85,139.65,140.32,164.28,165.75,172.19,173.61 .{ }^{1} \mathrm{H}$ NMR spectrum, and ${ }^{13} \mathrm{C}\left\{{ }^{1} \mathrm{H}\right\}$ (DEPT) spectra can be found in Figure $\mathbf{S 7}$ and Figure S8, respectively. Mass spectrometry (MALDI-TOF) $\mathrm{m} / \mathrm{z} 1009.1$ $\left(\left[\mathrm{M}^{+} \mathrm{H}\right]\right)$. Elemental analysis calcd. (\%) for $\mathrm{C}_{60} \mathrm{H}_{38} \mathrm{~N}_{4} \mathrm{O}_{4} \mathrm{Zn}_{2} \cdot 4 \mathrm{H}_{2} \mathrm{O} \mathrm{C} 66.62$, H 4.29, N 5.18 found C 66.58, H 4.00, N 5.94.

Transmission electron microscopy: TEM studies were carried out on a JEOL model JEM-1011 electron microscope with an acceleration voltage of $100 \mathrm{keV}, 50 \mu \mathrm{m} \mathrm{C} 2$ aperture, spot size 3 and variable dose rate. Analyzed liquid samples were prepared by dropcasting $70 \mu \mathrm{M}$ solutions of the corresponding BB 1 or 2 and/or $20 \mu \mathrm{g} / \mathrm{mL}$ of the MWCNT (DCM $99.8 \%$ 
purity as solvent) on formvar carbon film-covered square mesh copper grids and dried completely for $4 \mathrm{~h}$. Deviations from the latter concentrations are mentioned in the text. Solid film samples were embedded in an epoxybased resin, cured for $72 \mathrm{~h}$ at $80^{\circ} \mathrm{C}$, cut with a microtome and fixed on a copper double grid fixation system for analysis.

Scanning electron microscopy: Microscopic analysis was carried out using a JEOL microscope, model JSM-6400 at ambient temperature, work distance of $8 \mathrm{~mm}, 10^{-4}$ Torr pressure, beam voltage $10 \mathrm{kV}$ and variable probe current. Solid samples were fixed on standard sample holders without any previous treatment and directly analyzed at the microscope.

Dynamic light scattering: DLS analysis was performed using a Zetasizer Nano ZS (Malvern Instruments Ltd.) with $532 \mathrm{~nm}$ laser radiation source. Analytical procedure was carried out in all the cases with $1 \mathrm{~mL}$ of the corresponding solution on a standard fluorescence glass cuvette with $1 \mathrm{~cm}$ optical path.

Preparative solutions: Polymer films were obtained from preparative DCM solutions containing $10 \% \mathrm{~m} / \mathrm{v}$ high purity optical storage media grade bis-phenol-A polycarbonate provided by Bayer, the required BB 1 or $2(70 \mu \mathrm{M})$ and/or the multiwalled carbon nanotubes (>95\% purity, 100 $700 \mathrm{~nm}$ in length, 15-42 nm in external diameter, purchased at Nanocyl SA, Sambreville, Belgium). The amount of nanotubes:polymer ratio ranged between $10^{-4}: 99.9999$ to $5: 95$ in mass, where the systems which also contained one of the BBs alternatively included the last one in a ratio BB:polymer+nanotubes equal to $0.065: 99.935$ in mass. The solutions were prepared using anhydrous dichloromethane (99.8\%) purified, filtered and dried through a high-throughput solvent purification system.

Preparation of films: Films were prepared under high-efficiency particulate air-filtered laminar flow conditions following two different casting-from-solution strategies, depending on the nominal $d$ value required. Specimens with $d=100 \mu \mathrm{m}$ were prepared by casting preparative solutions at the required nanotube:polymer ratio on a closed injection spin-coating system. An aliquot of the solution (commonly $1 \mathrm{~mL}$ to $3 \mathrm{~mL}$, depending on the film thickness desired) was deposited on a circular flat aluminum mold $(6 \mathrm{~cm}$ diameter) fixed to the rotation system. The solution was then dispersed by spinning at $60 \mathrm{rpm}$ and dried at $25^{\circ} \mathrm{C}$ and $1 \mathrm{Atm}$. The films formed were dried at low vacuum (10-100 mBar), $25^{\circ} \mathrm{C}$ for $1-2 \mathrm{~h}$ and separated from the mold. Calibration of average $d$ was carried out using an Extramess 2000 inductive digital comparator (Mahr, Germany) with $0.1 \mu \mathrm{m}$ resolution and confirmed by SEM. Conversely, films with $d=10 \mu \mathrm{m}$ were prepared by casting preparative solutions on $50 \mu \mathrm{m}$ PET substrates and deposited over the last one using the doctor blade technique. An aliquot of the solution (commonly 1-5 mL, depending on the surface to cover and the film thickness desired) was deposited at the application region on the substrate and subsequently spread at $2.5 \mathrm{~mm} / \mathrm{s}$ to $30 \mathrm{~mm} / \mathrm{s}$ at $25{ }^{\circ} \mathrm{C}$ using a calibrated steel blade with sub-micrometer resolution adjustable height. Calibration of average $d$ was carried out adjusting the blade height and confirmed with the inductive digital comparator. The formed films were dried at low vacuum (10-100 mbar) and $25^{\circ} \mathrm{C}$ for $1-2 \mathrm{~h}$ and directly used after preparation. ITO films with $d=$ $130 \mathrm{~nm}\left(10^{-2} \mathrm{~S}\right)$ were provided by Sigma-Aldrich Chemie $\mathrm{GmbH}$ (Steinheim, Germany) supported on a $50 \mu \mathrm{m}$ thickness PET sheet; prior to use, ITO was subsequently cleaned with ethanol, dried at vacuum (10-100 mbar) and $25^{\circ} \mathrm{C}$ for $1-2 \mathrm{~h}$ and directly analyzed afterwards.

Electrical characterization of materials: Surface and volumetric electrical conductivity of solid films at conductivity values below $10^{-6} \mathrm{~S}$ (net readout) was determined with a Keithley Model 8009 high-resistivity test fixture with integrated Faraday box electrostatic shielding system coupled to a Keithley 6487 Picoammeter/Voltage source. Film samples with the dimension $6 \mathrm{~cm} \times 6 \mathrm{~cm}$ were directly analyzed within the fixture at applied voltages of $100 \mathrm{~V}$ and $500 \mathrm{~V}$ without any previous treatment. $\sigma_{\mathrm{v}}$ and $\sigma_{\mathrm{s}}$ of solid films at net conductivity readout above $10^{-6} \mathrm{~S}$ was determined following the two- and four-point probe method, using a Keithley High Performance Digital Multimeter model 2000 under automatic sweeping mode. Samples with the same abovementioned dimensions were pretreated at the measuring contact points with $4 \mathrm{~mm}$ width $/ 15 \mathrm{~mm}$ spacing thin layer strips of Electrodag 1415 silver ink supplied by Agar Scientific.

Optical characterization of films: UV-VIS-NIR measurements were carried out on a Lamba 1050 PerkinElmer spectrophotometer equipped with PMT, InGaAs and PbS detectors, double beam optics, double monochromator and D2 and W light sources. \% $\mathrm{T}_{550}$ optical transmission measurements were recorded on a Shimadzu UV1800 Spectrophotometer.

\section{Acknowledgements}

The Spanish Ministerio de Economía y Competitividad (CTQ2014-60419-R and the Severo Ochoa Excellence Accreditation 2014-2018, project SEV-2013-0319), the European Community Seventh Framework Program FP7-PEOPLE-ITN-2008 (grant number 238363), the DGAPA-PAPIIT program from National Autonomous University of Mexico (grant numbers IA205616 and IA205316), the National Council for Science and Technology from Mexico (CONACYT, grant number 251533), CERCA/Generalitat de Catalunya and ICREA are all acknowledged for support.

Keywords: dynamic self-assembly - hierarchical autoorganization - carbon nanotubes $\cdot$ polymer composites • conducting materials

[1] a) T. P. Bigioni, X.-M. Lin, T. T. Nguyen, E. I. Corwin, T. A. Witten, H. M Jaeger, Nat. Mater. 2006, 5, 265-270; b) M. Qian, A.C. Reber, A. Ugrinov, N. K. Chaki, S. Mandal, H. M. Saavedra, S. N. Khanna, S. Sen, P. S Weiss, ACS Nano 2010, 4, 235-240.

[2] a) A. Dong, J. Chen, P. M. Vora, J. M. Kikkawa, C. B. Murray, Nature 2010, 466, 474-477; b) B. G. Sumpter, L. Liang, A. Nicolaï, V. Meunier, Acc. Chem. Res. 2014, 47, 3395-3405; c) E. Y. S. Oliveira, R. Bode, M. V. Escárcega-Bobadilla, G. A. Zelada-Guillén, G. Maier, G. New J. Chem. 2016, 40, 4625-4634.

[3] a) X. Liu, Y.-Z. Long, L. Liao, X. Duan, Z. Fan, ACS Nano 2012, 6, 1888 1900; b) S. E. Mastroianni, T. H. Epps, Langmuir 2013, 29, 3864-3878; c) A. R. Chandrasekaran, H. Wady, H. K. K. Subramanian, Small 2016, 12, 2689-2700.

[4] K. J. M. Bishop, Angew. Chem. Int. Ed. 2016, 55, 1598-1600.

[5] A. H. Gröschel, A. Walther, T. I. Löbling, F. H. Schacher, H. Schmalz, A. H. E. Müller, Nature 2013, 503, 247-251.

[6] a) M. Brutschy, M. W. Schneider, M. Mastalerz, S. R. Waldvogel, Adv. Mater. 2012, 24, 6049-6052; b) M. Giese, L. K. Blusch, M. K. Khan, W Y. Hamad, M. J. MacLachlan, Angew. Chem. Int. Ed. 2014, 53, 88808884

[7] Y. Tian, X. Yan, M. L. Saha, Z. Niu, P. J. Stang, J. Am. Chem. Soc. 2016, 138, 12033-12036.

[8] a) C. F. J. Faul, Acc. Chem. Res. 2014, 47, 3428-3438; b) C. Zhang, P. Chen, H. Dong, Y. Zhen, M. Liu, W. Hu, Adv. Mater. 2015, 27, 5379-5387.

[9] a) H. Ma, J. Hao, Chem. Soc. Rev. 2011, 40, 5457-5471; b) S. A Majetich, T. Wen, R. A. Booth, ACS Nano 2011, 5, 6081-6084; c) S. Ilday, F. Ö. Ilday, R. Hübner, T. J. Prosa, I. Martin, G. Nogay, I. Kabacelik, Z Mics, M. Bonn, D. Turchinovich, H. Toffoli, D. Toffoli, D. Friedrich, B Schmidt, K.-H. Heinig, R. Turan, Nano Lett. 2016, 16, 1942-1948. 
[10] P. P. Edwards, A. Porch, M. O. Jones, D. V. Morgan, R. M. Perks, Dalton Trans. 2004, 2995-3002.

[11] D. Angmo, F. C. Krebs, J. Appl. Polym. Sci. 2013, 129, 1-14.

[12] a) A. Kumar, C. Zhou, ACS Nano 2010, 4, 11-14; b) K. Sakakibara, J. P. Hill, K. Ariga, Small 2011, 7, 1288-1308; c) K. Ellmer, Nat. Photonics 2012, 6, 809-817; d) C. J. M. Emmott, A. Urbina, J. Nelson, Sol. Energy Mater. Sol. Cells 2012, 97, 14-21; e) D. Jariwala, V. K. Sangwang, L. J. Lauhon, T. J. Marks, M. C. Hersam, Chem. Soc. Rev. 2013, 42, $2824-$ 2860 .

[13] a) J. K. Wassei, R. B. Kaner, Mater. Today 2010, 13, 52-59; b) M. F. L. De Volder, S. H. Tawfick, R. H. Baughman, A. J. Hart, Science 2013, 339 535-539.

[14] a) S. Roth, H. J. Park, Chem. Soc. Rev. 2010, 39, 2477-2483; b) K. Jiang, J. Wang, Q. Li, L. Liu, C. Li, S. Fan, Adv. Mater. 2011, 23, 1154-1161; c) D. S. Hecht, L. Hu, G. Irvin, Adv. Mater. 2011, 23, 1482-1513; d) X. Sun, T. Chen, H. Peng, Acc. Chem. Res. 2013, 46, 539-549; e) J. Du, S. Pei, L. Ma, H.-M. Cheng, Adv. Mater. 2014, 26, 1958-1991; f) M. Layani, A Kamyshny, S. Magdassi, Nanoscale 2014, 6, 5581-5591; g) J. Li, G. P. Pandey, Annu. Rev. Phys. Chem. 2015, 66, 331-356;.

[15] S. H. Kim, C. S. Haines, N. Li, K. J. Kim, T. J. Mun, C. Choi, J. Di, Y. J. Oh, J. P. Oviedo, J. Bykova, S. Fang, N. Jiang, Z. Liu, R. Wang, P. Kumar R. Qiao, S. Priya, K. Cho, M. Kim, M. S. Lucas, L. F. Drummy, B. Maruyama, D. Y. Lee, X. Lepró, E. Gao, D. Albarq, R. Ovalle-Robles, S. J. Kim, R. H. Baughman, Science 2017, 357, 773-778.

[16] L. Hu, D. S. Hecht, G. Grüner, Nano Lett. 2004, 4, 2513-2517.

[17] a) P. N. Nirmalraj, P. E. Lyons, S. De, J. N. Coleman, J. J. Boland, Nano Lett. 2009, 9, 3890-3895; b) M. Y. Timmermans, D. Estrada, A. G.
Nasibulin, J. D. Wood, A. Behnan, D. Sun, Y. Ohno, J. W. Lyding, E. P. Hassanien, E. Kauppinen, Nano Res. 2012, 5, 307-319.

[18] Z. Yang, M. Liu, C. Zhang, W. W. Tjiu, T. Liu, H. Peng, T. Liu, Angew. Chem. Int. Ed. 2013, 52, 3996-3999.

[19] M. V. Escárcega-Bobadilla, G. A. Zelada-Guillén, S. V. Pyrlin, M. Wegrzyn, M. M. D. Ramos, E. Giménez, A. Stewart, G. Maier, A. W. Kleij, Nat. Commun. 2013, 4, 2648.

[20] a) S. Akine, M. Miyashita, T. Nabeshima, J. Am. Chem. Soc. 2017, 139, 4631-4634; b) P. D. Frischmann, S. Guieu, R. Tabeshi, M. J. MacLachlan, J. Am. Chem. Soc. 2010, 132, 7668-7675; c) S. J. Wezenberg, G. Salassa, E. C. Escudero-Adán, J. Benet-Buchholz, A. W. Kleij, Angew. Chem. Int. Ed. 2011, 50, 713-716; d) G. Salassa, M. J. J. Coenen, S. J Wezenberg, B. L. M. Hendriksen, S. Speller, J. A. A. W. Elemans, A. W. Kleij, J. Am. Chem. Soc. 2012, 134, 7186-7192; e) G. Consiglio, I. Pietro Oliveri, S. Failla,S. Di Bella, Inorg. Chem. 2016, 55, 10320-10328; f) G. Consiglio, S. Failla, P. Finocchiaro, I. Pietro Oliveri, S. Di Bella, Inorg Chem. 2012, 51, 8409-8418; g) M. Mastalerz, H.-J. S. Hauswald, R. Stoll, Chem. Commun. 2012, 48, 130-132.

[21] J. K. Sprafke, S. D. Stranks, J. H. Warner, R. J. Nicholas, H. L. Anderson, Angew. Chem. Int. Ed. 2011, 50, 2313-2316.

[22] R. Gordon, MRS Bulletin 2000, 25, 52-57.

[23] A. Kämpfe, E. Kroke, J. Wagler, Eur. J. Inorg. Chem. 2009, 1027-1035.

[24] H.-C. Zhang, W.-S. Huang, L. Pu, J. Org. Chem. 2001, 66, 481-487.

[25] C. Görl, H. G. Alt, J. Organomet. Chem. 2007, 692, 5727-5753.

[26] M. V. Escárcega-Bobadilla, G. Salassa, M. Martínez Belmonte, E. C. Escudero-Adán A. W. Kleij, Chem.-Eur. J. 2012, 18, 6805-6810. 


\section{Entry for the Table of Contents}

\section{FULL PAPER}

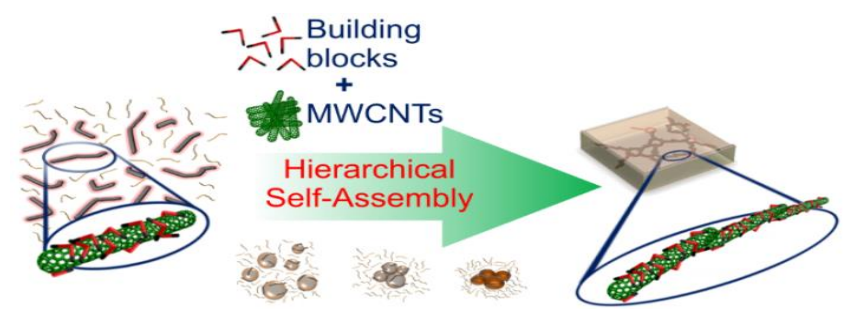

Putting things to order: Nanoscale anisotropic supramolecular interactions between carbon nanotubes and molecular building blocks based on bimetallic $\mathrm{Zn}$ (salen) complexes allow for hierarchical self-assembly control at the microscale to produce mesoscale electrical percolation networks, which enhances macroscale conductivity in polymer composites up to six orders of magnitude.
Gustavo A. Zelada-Guillén, * Martha V. Escárcega-Bobadilla, * Marcin Wegrzyn Enrique Giménez, Gerhard Maier and $\operatorname{Arjan}$ W. Kleij*

Page No. - Page No.

Enhanced Conductivity for Carbon Nanotube based Materials through Supramolecular Hierarchical SelfAssembly 